\title{
A Factorization Approach to Evaluating Simultaneous Influence Diagrams
}

\author{
Weihong Zhang and Qiang Ji, Senior Member, IEEE
}

\begin{abstract}
Evaluating an influence diagram (ID) is a challenging problem because its complexity increases exponentially in the number of decision nodes in the diagram. In this paper, we examine the problem for a special class of IDs where multiple decisions must be made simultaneously. We describe a brief theory that factorizes out the computations common to all policies in evaluating them. Our evaluation approach conducts these computations once and uses them across all policies. We identify the ID structures for which the approach can achieve savings. We show that the approach can be used to efficiently recompute the optimal policy of an ID when its structure or parameters change. Finally, we demonstrate the superior performance of the approach by simulation studies and a military planning example.
\end{abstract}

Index Terms-Algorithm, decision making under uncertainty, graphical model, influence diagram (ID), military analysis.

\section{INTRODUCTION}

A $\mathrm{N}$ INFLUENCE diagram (ID) is a plausible graphical model for decision making under uncertainty [1]. An ID comprises of decision nodes, random nodes, value nodes, and the probabilistic relations among these nodes. An ID is a more compact representation of a decision tree, which is a simple tool for decision analysis [2].

Given an ID, a policy prescribes an action choice for each decision node. Evaluating a policy is to compute the expected value of the ID under the policy. Evaluating an ID is to find the optimal policy that maximizes the expected value of the ID. A generic approach to evaluating an ID has to enumerate all policies, compare the expected utilities under them, and choose the optimal one. However, the number of policies grows exponentially with the number of decision nodes. This renders the approaches for general ID evaluation very inefficient and infeasible for large IDs. Consequently, it is advisable to study efficient algorithms for special IDs.

Most of the previous approaches assume that there exists a linear ordering among the decision nodes. This ordering implies that the choice of a decision node is known to the decision maker when he/she chooses the actions for the successive decision nodes (e.g., see [13]). For a decision node, this linear

Manuscript received February 19, 2004; revised July 20, 2004 and December 22, 2004. The work was supported in part by the Air Force Office of Scientific Research (AFOSR) under Grant F49620-03-1-0160. Part of the work for this project was also supported by the Air Force Research Laboratory (AFRL)/Rome summer visiting faculty program. This paper was recommended by Associate Editor Yang.

The authors are with the Department of Electrical, Computer and Systems Engineering, Rensselaer Polytechnic Institute, Troy, NY 12180-3590 USA.

Digital Object Identifier 10.1109/TSMCA.2005.855753 ordering usually can be exploited to decompose the ID into one fraction prior to the node and the other fraction posterior to the node. The choice for the decision node can be made using the fraction posterior to the node. The procedure repeats for each decision node.

In this paper, we examine the ID evaluation problem for a special class of IDs in which decision nodes have no parents. Essentially, an ID with this property assumes no precedence relationship among decision nodes. In other words, one has to determine the choices for all decision nodes simultaneously. For this reason, such an ID is said to be simultaneous. The simultaneity assumption prevails in real-world problem domains. For instance, a military planner must select among a number of available actions to achieve his/her overall goal success; a business owner must consider multiple elements in order to maximize his/her monetary profit.

In evaluating a simultaneous ID, we exploit the assumption and divide the ID into two fractions, calling them the upstream and downstream. Roughly, the upstream consists of decision nodes and their children nodes through which the decisions propagate their impacts on the ID. Informally, these child nodes are called interface nodes. The downstream consists of the interface nodes and their succeeding nodes. We present a representation theorem, showing that the expected value of a value node under a policy can be represented as the sum of some intermediate quantities weighted by the probabilities determined by the policy. These intermediate quantities involve only the downstream. The factorization approach we proposed computes them once but uses them across all policies. The computational gain brought by the approach depends on the size of the downstream. Usually, larger downstream size implies more savings.

We organize the paper as follows. In the next section, we discuss related work to this research. We then introduce IDs and the evaluation problem. In Section IV, we describe the representation theorem and develop the factorization approach. In Section V, we discuss two extensions of the approach: how it can be adapted to network structure/parameter changes and how it can be used in planning over time. We report empirical results on simulation studies and a military planning example in Section VI. Finally, we conclude the paper in Section VII.

\section{RELATED WORK}

Since IDs were introduced by Howard and Matheson [1], a variety of approaches have been proposed to find the optimal policy of a given ID. To mitigate the exponential growth 
problem of the policy number in the number of decision nodes, researchers have studied several special ID classes and proposed efficient approaches exploiting their specific problem characteristics. We give a brief survey of these IDs and their solutions.

\section{A. Regular and No-Forgetting IDs}

To some extent, most IDs that have been studied assume a precedence ordering of the decision nodes. A regular ID assumes that there is a directed path containing all decision nodes; a no-forgetting ID assumes that each decision node and its parents are also parents of the successive decision nodes; and a stepwise decomposable ID assumes that the parents of each decision node divide the ID into two separate fractions. These assumptions are different from ours, which requires the actions to be chosen simultaneously. There exist direct and indirect approaches evaluating a regular no-forgetting ID. A direct approach works on the ID and evaluates it directly. Shachter [3] proposed an algorithm that evaluates an ID by applying a series of value-preserving reductions. A value-preserving reduction is an operation that can transform an ID into another one with the same expected value. Specifically, Shachter identified the following four reductions arc reversal, barren-node removal, random-node removal, and decision-node removal. An indirect approach first transforms an ID into an intermediate structure whose optimal policy (or value) remains the same as in the original ID. It then evaluates the intermediate structure and obtains the optimal policy. For instance, Howard and Matheson discussed a way to transform an ID into a decisiontree network and to compute an optimal policy from the decision tree. In transforming an ID into a decision-tree network, a basic operation is arc reversal [1], [3]. Since a no-forgetting ID must be stepwise decomposable, stepwise decomposability is more general than no-forgetting.

In most ID evaluation approaches, the ordering of decision nodes is an important information source in decision making and therefore, is exploited to evaluate the optimal decision for decision nodes [4]-[6]. A stepwise decomposable ID can be evaluated by a divide-and-conquer approach. The approach deals with one decision node at a time [7]. For each decision node, its parental set separates an ID into two parts-a body and a tail. The tail is a simple ID with only one decision node. The body's value node is a new value node whose value function is obtained by evaluating the tail. In evaluating a stepwise decomposable ID, the approach begins with a leaf decision node and repeats the decomposition/evaluation procedure for the preceding decision nodes. In evaluating the tail with only one single decision node, the problem is reduced to that of computing posterior probabilities in a Bayesian network. Hence, the approach uses probabilistic inference techniques to evaluate an ID. Cooper [8] initiated the research in this direction. He gave a recursive formula for computing the maximal expected utilities and optimal policies of IDs. Shachter and Peot [9] showed that the problem of ID evaluation can be reduced to a series of probabilistic inferences. Zhang [13] described an algorithm that induces much easier probabilistic inferences than those in [8] and [9].

\section{B. Partial IDs}

There also exists research work that relaxed the regularity or no-forgetting assumption. The specific ID types include partial IDs, unconstrained IDs and limited memory ID (LIMID), which is a compact representation of IDs. A partial ID is an ID that allows a non-total ordering of decision nodes [10]. Because the solution to a partial ID depends on the temporal ordering of the decisions, it is of interest to find the conditions identifying a class of partial IDs whose solution is independent of the legal evaluation ordering. Based on the concept of d-connectivity, Nielsen and Jensen presented an algorithm determining whether or not a partial ID represents well-defined scenarios, and they also addressed the problem of whether all admissible orderings yield the same optimal strategy.

An unconstrained ID is an ID where the order of decision nodes and the observable random nodes is not determined [11]. For an unconstrained ID, it is of interest to determine the order of decision nodes and information on which set of nodes is necessary for decision making in a decision node. For this purpose, a set of rules have been developed in order to determine the choice of the next decision node, given the current information. Such a decision choice may be dependent on the specific information from the past.

Another recently proposed ID is called LIMID, which violates the no-forgetting assumption [12]. In contrast to the regular and no-forgetting assumption, the assumption behind a LIMID is that only requisite information for the computation of optimal policies is depicted in the graphical representation. Two properties pertaining to LIMIDs are: 1) any ID can be converted to a LIMID; and 2) the converted LIMID is more compact than the original ID in the sense that only requisite information is depicted in the LIMID for computing an optimal policy. By these properties, one may convert an ID to its LIMID version and solve the LIMID instead of the original ID. This optimal policy is also optimal in the original ID. The algorithm solving a LIMID exploits the fact that the entire decision problem can be partitioned into a set of smaller decision problems, each of which has one decision node only. This is analogous to the divide-and-conquer approach [13].

\section{Simultaneous IDs}

From its root definition, an ID does not impose a precedence ordering of the decision nodes. As an example, there are military applications that need to choose multiple actions simultaneously. A simultaneous ID is suitable for this situation. We exploit this assumption and divide a simultaneous ID into the upstream and the downstream fractions. The decomposition takes the random and value nodes as interface nodes between the upstream and the downstream. The computations involving the downstream fraction can be precomputed and reused across all policies in evaluating them. This computation-sharing schema can greatly accelerate the procedure of finding the optimal policy for a given ID, as indicated in our theoretical and empirical analysis.

Technically, the factorization approach has some conceptual similarities to the probabilistic inference-based algorithm [13]. 
Both algorithms divide the ID into two fractions. However, there are apparent differences. In [13], the separation of an ID relies on a single decision node. With respect to a decision node, roughly, the body contains the predecessors of the decision nodes, while the tail contains the successors. The choice of the decision node is evaluated by the tail part. This is quite different from our factorization approach, where the separation relies on the set of interface nodes. The set of the interface nodes separates an ID into two fractions: roughly, the upstream contains the predecessors of all interface nodes, while the downstream contains the successors. This difference in solution techniques stems from the difference in assumption-the probabilistic inference algorithm works with a regular ID that specifies a linear order among decision nodes, whereas the factorization algorithm works with a simultaneous ID that assumes no ordering among decision nodes.

\section{INFLUENCE DIAGRAM}

Mathematically, an ID $\mathcal{I}$ is a directed acyclic graph consisting of three types of nodes and the links among these nodes [1].

1) Its node set is partitioned into a set of random nodes $\mathcal{Y}$, a set of decision nodes $\mathcal{X}$, and a set of value nodes $\mathcal{U}$. A value node cannot have children. The links characterize the conditional dependence among the nodes in the ID. Specifically, links to a random node indicate the probabilistic dependence of the node on its parents; links to a decision node indicate the information available to the planner at the time the planner must choose a decision for it; and links to a value node indicate the functional dependencies.

We will adopt the following notational conventions. We will use bold-typed letters such as $\mathcal{Z}$ to denote a set of variables and capital letters such as $Z$ to denote a variable in the set. Each random or decision node $Z$ is associated with a set $\Omega_{Z}$, denoting the set of its possible states. The set $\Omega_{Z}$ is called the domain of node $Z$. An element in $\Omega_{Z}$ is denoted by a low-case letter $z$. For any node $Z$, we use $\pi(Z)$ to denote its parent set. For any subset $\mathcal{Z}^{\prime} \subset \mathcal{Y} \cup \mathcal{X}$, we use $\Omega_{\mathcal{Z}^{\prime}}$ to denote the Cartesian product $\Pi_{Z \in \mathcal{Z}^{\prime}} \Omega_{Z}$. For convenience, we shall interchangeably use a node and a variable. Without loss of generality, we assume that all the nodes are binary throughout this paper.

2) For each decision or random node $Z$, given an assignment of $\pi(Z)$, the distribution $P(Z \mid \pi(Z))$ specifies the probability of $Z$ being in each state of the node $Z$. Such a distribution is called a conditional probability table (CPT) in the case that the domain of the variable $Z$ is a finite set.

3) For each value node $U, g_{U}$ is a value function $g_{U}$ : $\Omega_{\pi(U)} \rightarrow R$, where $R$ denotes the set of the real numbers.

To avoid unnecessary notations, we define the (optimal) policy concept only for a simultaneous ID. ${ }^{1}$ A policy, denoted by $\delta$, specifies one action choice for each decision node in $\mathcal{X}$. Hence, a policy $\delta$ can be denoted by $\left(\delta_{1}, \ldots, \delta_{n}\right)$, where $\delta_{i}$ belongs to the domain of $X_{i}$ for each $i$.

\footnotetext{
${ }^{1}$ For general IDs, the definition of an (optimal) policy can be found in, e.g., [6].
}

Given a policy $\delta$, a probability $P_{\delta}$ can be defined over the random nodes and decision nodes as follows:

$$
P_{\delta}(\mathcal{Y}, \mathcal{X})=\Pi_{Y \in \mathcal{Y}} P(Y \mid \pi(Y)) \Pi_{i=1}^{n} P_{\delta}\left(X_{i}\right)
$$

where $P(Y \mid \pi(Y))$ is specified in the definition of $\mathcal{I}$, while $P_{\delta}\left(X_{i}\right)$ is equal to 1.0 if $X_{i}=\delta_{i}$, and 0.0 otherwise.

The expectation of the value node $U$ under policy $\delta$, denoted by $E_{\delta}[U]$, is defined as

$$
E_{\delta}[U]=\sum_{\pi(U)} P_{\delta}(\pi(U)) g_{U}(\pi(U)) .
$$

The expected value $E_{\delta}$ of $\mathcal{I}$ under the policy $\delta$ is the sum $E_{\delta}[U]$ over all value nodes $U$ in $\mathcal{U}$, i.e.,

$$
E_{\delta}=\sum_{U \in \mathcal{U}} E_{\delta}[U]
$$

For simplicity, $E_{\delta}$ is also called the expected value of policy $\delta$. Evaluating a policy $\delta$ means to compute its expected value. The maximum of $E_{\delta}$ over all policies is the optimal (expected) value of $\mathcal{I}$. An optimal policy is the policy that achieves the optimal expected value. To evaluate an ID is to find an optimal policy and to compute its optimal expected value.

\section{THE FACTORIZATION APPROACH}

In this section, we describe the representation theorem and the factorization approach.

\section{A. The Idea}

From its definition, an ID is a network structure consisting of decision nodes, random nodes, and value nodes. Among them, in determining the expected value of the ID, a decision node plays a different role from a random or a value node. The choices of a decision node can affect the expected value of the ID through changing the CPTs of its child random nodes, or through changing the value functions of its child value nodes (note that a decision node cannot have another decision node as child in a simultaneous ID). In this sense, a node, if it is a child of a decision node, serves as an interface through which the choices of decision nodes may affect the value of the ID. Such a node is called an interface node. All interface nodes constitute an interface set. Collectively, an interface set serves as an interface of an ID through which policies can affect the expected value of the ID. Consequently, an ID can be divided into two fractions: the upstream fraction, which includes the interface nodes and the nodes "preceding" them, and the downstream fraction, which includes the interface nodes and the nodes "succeeding" the interface nodes.

Example: We use the ID in Fig. 1 to informally illustrate these concepts. The ID has two decision nodes $\left\{X_{1}, X_{2}\right\}$, five random nodes $\{A, B, C, D, H\}$, and one value node $U$. The interface set $\mathcal{Y}_{\text {in }}$ is $\{A, C\}$ since they have parental decision nodes. The upstream is $\left\{X_{1}, X_{2}, A, B, C\right\}$, which consists of two interface nodes $A$ and $C$, node $X_{1}$ preceding node $A$, and nodes $B$ and $X_{2}$ preceding node $C$. The downstream is 


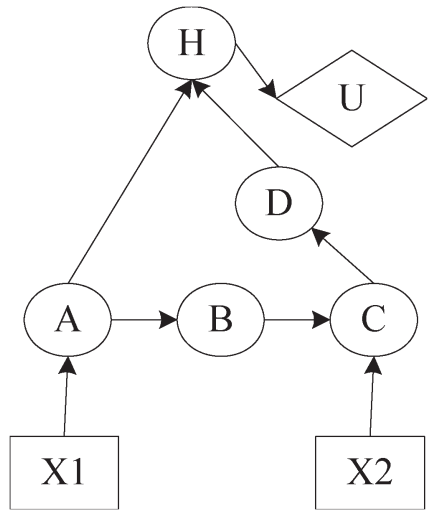

Fig. 1. ID to illustrate the representation theorem.

$\{A, C, H, D, U\}$, which consists of interface nodes $A$ and $C$, the nodes $H, D, U$ succeeding to the interface nodes.

Interestingly, corresponding to the structural separation that an ID can be divided into two fractions, the expected value of a value node under a policy breaks into two fractions, each of which involving only the upstream or the downstream of the ID.

\section{B. The Theorem}

We formalize the above idea in this section. For the sake of simplicity, throughout the paper, unless explicitly stated, we assume that: 1) the ID has only one value node; and 2) the value node has no decision node as its parent. We also note that our results in this paper generalize to the IDs with multiple value nodes and with value nodes having parental decision nodes. We relax these assumptions at the end of this section.

We begin by defining several concepts. A random node $Y$ is an interface node if its parent set has at least one decision variable, i.e., $\pi(Y) \cap \chi \neq \emptyset$. The interface set of an ID is the set of all interface nodes. Due to the above assumptions, the interface set contains only random nodes; for this reason, we denote the set by $\mathcal{Y}_{\text {in }}$. The upstream of the ID includes the interface set and all ancestors of the nodes in the interface. By this definition, in addition to the interface random nodes and decision nodes, the upstream may contain the random-node ancestors of the interface nodes. These ancestral nodes must be included because they, together with decision nodes, determine the CPTs of the interface nodes. These ancestral random nodes form a set denoted by $\mathcal{Y}_{0}$.

Given an ID, we can efficiently identify its upstream using a queuing mechanism. We initialize a queue to be the interface set $\mathcal{Y}_{\text {in }}$ (it can be readily built by checking whether there is a parental decision node for every node in the ID) and the upstream $\mathcal{I}_{\text {up }}$ to be empty. At each step, a node is removed from the queue and added to $\mathcal{I}_{\text {up }}$ if it is not in $\mathcal{I}_{\text {up }}$. The parents of the node, if not present in $\mathcal{I}_{\text {up }}$ thus far, are added to the queue. The procedure terminates when the queue is empty. When it terminates, the set $\mathcal{I}_{\text {up }}$ becomes the upstream set. The procedure must terminate after a finite number of steps because an ID is a directed acyclic graph.

The upstream can be partitioned into three sets: the set $\mathcal{X}$ of decision nodes, interface set $\mathcal{Y}_{\text {in }}$, and the set $\mathcal{Y}_{0}$ of randomnode ancestors of interface nodes. Given a policy $\delta$, we define a function $f_{\delta}$ from $\Omega_{y_{\text {in }}}$ to the real line $R$. For notations, we let $m$ be the number of nodes in the set $\mathcal{Y}_{\mathrm{in}}, Y_{\mathrm{in}}^{1: m}$ be a short notation of $\left\{Y_{\mathrm{in}}^{1}, \ldots, Y_{\mathrm{in}}^{m}\right\}$, and $y_{\mathrm{in}}^{1: m}$ be an assignment to all interface variables, i.e., an element of $\Omega_{Y_{\mathrm{in}}^{1: m}}$

$$
f_{\delta}\left(y_{\mathrm{in}}^{1: m}\right)=\sum_{Y \in \mathcal{Y}_{0}} \Pi_{Y \in \mathcal{Y}_{0} \cup \mathcal{Y}_{\mathrm{in}}} P_{\delta}(Y \mid \pi(Y)) \Pi_{i=1}^{n} P_{\delta}\left(X_{i}\right)
$$

where $\Pi_{Y \in \mathcal{Y}_{0} \cup \mathcal{Y}_{\text {in }}} P_{\delta}(Y \mid \pi(Y)) \Pi_{i=1}^{n} P_{\delta}\left(X_{i}\right)$ is the joint probability distribution of the variables in $\mathcal{X}, \mathcal{Y}_{0}$, and $\mathcal{Y}_{\text {in }}$, given policy $\delta$. Hence, $f_{\delta}\left(Y_{\mathrm{in}}^{1: m}\right)$ is the conditional probability that the interface $Y_{\text {in }}^{1: m}=y_{\text {in }}^{1: m}$ occurs upon the policy $\delta$. For convenience, we call them interface probabilities.

In contrast to the upstream, the downstream of an ID is the set consisting of all the interface nodes and their descendants. The downstream contains the value node, the interface nodes, and the random nodes that do not belong to the upstream. We use $\mathcal{Y}_{1}$ to denote the set of noninterface random nodes in the downstream. Note that the random nodes in the interface set belong to both the upstream and the downstream. For an assignment $y_{\mathrm{in}}^{1: m}$ of the set $Y_{\mathrm{in}}^{1: m}$ and the value node $U$, we can define a function as follows;

$$
f_{\mathrm{in}, U}\left(y_{\mathrm{in}}^{1: m}\right)=\sum_{Y \in \mathcal{Y}_{1}} \Pi_{Y \in \mathcal{Y}_{1}} P_{\delta}(Y \mid \pi(Y)) g_{U}(\pi(U)) .
$$

To see that $f_{\mathrm{in}, U}$ is a function of $Y_{\mathrm{in}}^{1: m}$, we note that the interface variables may appear in $\pi(Y)$ for $Y \in \mathcal{Y}_{1}$. Given an assignment $y_{\mathrm{in}}^{1: m}$ of $Y_{\mathrm{in}}^{1: m}, f_{\mathrm{in}, U}\left(y_{\mathrm{in}}^{1: m}\right)$ is the expected utility conditioned on the assigned interface $y_{\mathrm{in}}^{1: m}$. These quantities are called interface utilities for convenience. Since $\pi(Y)$ for $Y$ in $\mathcal{Y}_{1}$ must belong to the downstream, $P_{\delta}(Y \mid \pi(Y))$ is independent of policy $\delta$. Consequently, these utilities are independent of policy $\delta$.

Theorem 1: Given a policy $\delta$ and a value node $U$, the expected value of the node $U$ under policy $\delta$

$$
E_{\delta}[U]=\sum_{y_{\mathrm{in}}^{1: m} \in \Omega_{Y_{\mathrm{in}}^{1: m}}} f_{\delta}\left(y_{\mathrm{in}}^{1: m}\right) \cdot f_{\mathrm{in}, U}\left(y_{\mathrm{in}}^{1: m}\right)
$$

Proof: We show that $E_{\delta}[U]$ can be rewritten as the sum of the interface utility $f_{\mathrm{in}, U}$ weighted by the probability $f_{\delta}$ over all interfaces

$$
\begin{aligned}
E_{\delta}[U]= & \sum_{\pi(U)} P_{\delta}(\pi(U)) g_{U}(\pi(U)) \\
= & \sum_{\pi(U)}\left[\sum_{\frac{\mathcal{V}}{\pi(U)}} \Pi_{Y \in \mathcal{Y}} P(Y \mid \pi(Y)) \Pi_{i=1}^{n} P_{\delta}\left(X_{i}\right)\right] \\
& \times g_{U}(\pi(U)) \\
= & \sum_{Y \in \mathcal{Y}_{\text {in }}} \sum_{Y \in \mathcal{Y}_{0}} \sum_{Y \in \mathcal{Y}_{1}} \Pi_{Y \in \mathcal{Y}_{0} \cup \mathcal{Y}_{\mathrm{in}}} P(Y \mid \pi(Y)) \\
& \times \Pi_{Y \in \mathcal{Y}_{1}} P(Y \mid \pi(Y)) \Pi_{i=1}^{n} P_{\delta}\left(X_{i}\right) g_{U}(\pi(U))
\end{aligned}
$$




$$
\begin{aligned}
& =\sum_{Y \in \mathcal{Y}_{\text {in }}}\left[\sum_{Y \in \mathcal{Y}_{0}} \Pi_{Y \in \mathcal{Y}_{0} \cup \mathcal{Y}_{\mathrm{in}}} P(Y \pi(Y))\right] \\
& \times\left[\sum_{Y \in \mathcal{Y}_{1}} \Pi_{Y \in \mathcal{Y}_{1}} P(Y \mid \pi(Y)) g_{U}(\pi(U))\right] \\
& =\sum_{y_{\mathrm{in}}^{1: m} \in \Omega_{Y_{\mathrm{in}}^{1: m}}} f_{\delta}\left(y_{\mathrm{in}}^{1: m}\right) \cdot f_{\mathrm{in}, U}\left(y_{\mathrm{in}}^{1: m}\right) .
\end{aligned}
$$

Step (a) is true by (2). At step (b), $\mathcal{Y} / \pi(U)$ is the difference set of $\mathcal{Y}$ and $\pi(U)$. This step is true by inserting (1) into (2). Step (c) follows from the fact that $\{\pi(U), \mathcal{Y} / \pi(U)\}$ and $\left\{\mathcal{Y}_{0}, \mathcal{Y}_{\text {in }}, \mathcal{Y}_{1}\right\}$ are two partitions of the set $\mathcal{Y}$. At step (d), we break the distribution $\Pi_{Y \in \mathcal{Y}} P(Y \mid \pi(Y)) \times$ $\Pi_{i=1}^{n} P_{\delta}\left(X_{i}\right)$ into two fractions $\Pi_{Y \in \mathcal{Y}_{0} \cup \mathcal{Y}_{\text {in }}} P(Y \mid \pi(Y))$ and $\Pi_{Y \in \mathcal{Y}_{1}} P(Y \mid \pi(Y)) g_{U}(\pi(U))$. At step (e), we replace the two fractions with the definitions of the interface utilities and interface probabilities.

By the theorem, given a policy $\delta$ and a value node $U$, the expected value of the node under the policy can be represented as the sum of the multiplications of the interface utilities and corresponding interface probabilities.

Example (Continued): For the ID in Fig. 1, we show how to represent $E_{\delta}[U]$ for the value node $U$ and a given policy $\delta$. Let the policy $\delta$ be $\left(\delta_{1}, \delta_{2}\right)$, where $\delta_{i}$ is the decision choice of $X_{i}$ for $i=1,2$. By definition

$$
\begin{array}{rl}
E_{\delta}[U]=\sum_{H} \sum_{A B C D} & P\left(A \mid \delta_{1} \delta_{2}\right) P(B \mid A) P\left(C \mid B \delta_{2}\right) \\
& \times P(D \mid C) P(H \mid A D) \Pi_{i=1}^{2} P_{\delta}\left(X_{i}\right) g_{U}(H) .
\end{array}
$$

The two functions are defined as follows.

$$
\begin{aligned}
f_{\delta}(A, C) & =\sum_{B} P\left(A \mid \delta_{1} \delta_{2}\right) P(B \mid A) P\left(C \mid B \delta_{2}\right) \Pi_{i=1}^{2} P_{\delta}\left(X_{i}\right) \\
f_{\mathrm{in}, U}(A, C) & =\sum_{H D} P(H \mid A D) P(D \mid C) g_{U}(H) .
\end{aligned}
$$

It can be verified that $E_{\delta}[U]=\sum_{A, C} f_{\delta}(A, C) \cdot f_{\mathrm{in}, U}(A, C)$.

We examine the assumptions we made at the beginning of this section. First, we have assumed that there is only one value node. In case of multiple value nodes, we may apply the representation theorem to each node. The expected value of a policy is the additive sum of the expected values of all value nodes under the policy.

Second, we have assumed that the value node has no decision nodes as its parents. In the other case that the value node has a decision node as its parents, the functions $f_{\delta, U}$ and $f_{\text {in }}$ can be defined as follows, such that the theorem holds

$$
\begin{aligned}
f_{\delta, U}\left(Y_{\mathrm{in}}^{1: m}\right)= & \sum_{Y \in \mathcal{Y}_{0}} \Pi_{Y \in \mathcal{Y}_{0} \cup \mathcal{Y}_{\text {in }}} P_{\delta}(Y \mid \pi(Y)) \\
& \times \Pi_{i=1}^{n} P_{\delta}\left(X_{i}\right) g_{U}(\pi(U)) \\
f_{\text {in }}\left(Y_{\mathrm{in}}^{1: m}\right)= & \sum_{Y \in \mathcal{Y}_{1}} \Pi_{Y \in \mathcal{Y}_{1}} P_{\delta}(Y \mid \pi(Y)) .
\end{aligned}
$$

TABLE I

FACTORIZATION APPROACH TO ID EVALUATION

1. Pre-compute the quantities $f_{i n, U}\left(y_{i n}^{1: m}\right)$ for all $y_{i n}^{1: m}$ in $\Omega_{Y_{i n}^{1: m}}$

2. For each policy $\delta$

2.1 compute $f_{\delta}\left(y_{i: m}^{1: m}\right)$ for each assignment of $Y_{i n}^{1: m}$

2.2 compute $E_{\delta}[U]$ by Equation (6)

3. Return the policy that maximizes $E_{\delta}[U]$

Therefore, we can lift the assumption that the value node has no decision node as parents. In this case, $U$ is also called an interface node, but it belongs to the upstream only. The reason is that, by definition, a value node cannot have children and therefore cannot produce impact on the downstream. ${ }^{2}$ Note that $f_{\delta}$ changes to $f_{\delta, U}$, since the value node is considered in computing the quantities relevant to the upstream. Interestingly, it can be proven that $f_{\text {in }}(=1.0)$ is a constant. To see why, let us assume that the size of $\mathcal{Y}_{1}$ be $k$. We enumerate the set $\mathcal{Y}_{1}$ as $\left\{Y_{1}^{1}, \ldots, Y_{1}^{k}\right\}$ such that a node's parents appear after the node in the set. In computing $f_{\text {in }}\left(Y_{\text {in }}^{1: m}\right)$, we can sequentially sum out the variables in $\mathcal{Y}_{1}$ in the enumerated order. Ultimately, we have $f_{\text {in }}=1.0$.

\section{The Algorithm}

By the representation theorem, the expected value of a policy is represented as the sum of interface utilities weighted by the corresponding interface probabilities. The interface utilities are independent of the individual policies, whereas the interface probabilities are dependent on the policies. Therefore, the interface utilities can be factored out, i.e., they can be calculated once and reused across all the policies.

This is the idea behind our factorization approach, which is described in Table I. The factored-out computations are calculated once at line 1 . They are used for all policies at line 2.2. Note that the procedure generalizes to IDs with multiple value nodes.

\section{Complexity Analysis}

It is of interest to compare the approach and the generic brute-forced approach that evaluates a policy directly by combining (1) and (2). Let $n$ be the number of decision nodes. Thus, the size of the policy space is $2^{n}$. Let the complexity of evaluating one policy be $C$. The complexity $C$ breaks into three pieces: computing $f_{\delta}$, computing $f_{\mathrm{in}, U}$, and computing $E_{\delta}[U]$ by (6). We denote them, respectively, by $C_{1}, C_{3}$, and $C_{2}$. To evaluate all policies, the generic approach has complexity $2^{n} C$, i.e., $2^{n}\left(C_{1}+C_{2}+C_{3}\right)$. In contrast, since the factorization approach computes $f_{\mathrm{in}, U}$ only once but uses them $2^{n}$ times, its complexity is $2^{n}\left(C_{1}+C_{2}\right)+C_{3}$. A good measure to predict the computational gain is the size of the downstream, i.e., the number of nodes in it. In one extreme, if the downstream contains only the interface nodes and the value nodes (thus, $C_{3}$ is a constant), the two approaches have the same complexity. In the other extreme, if the downstream contains far more nodes

\footnotetext{
${ }^{2}$ Note that this is different from random nodes having parental decision nodes. Such a random node belongs to both the upstream and the downstream.
} 


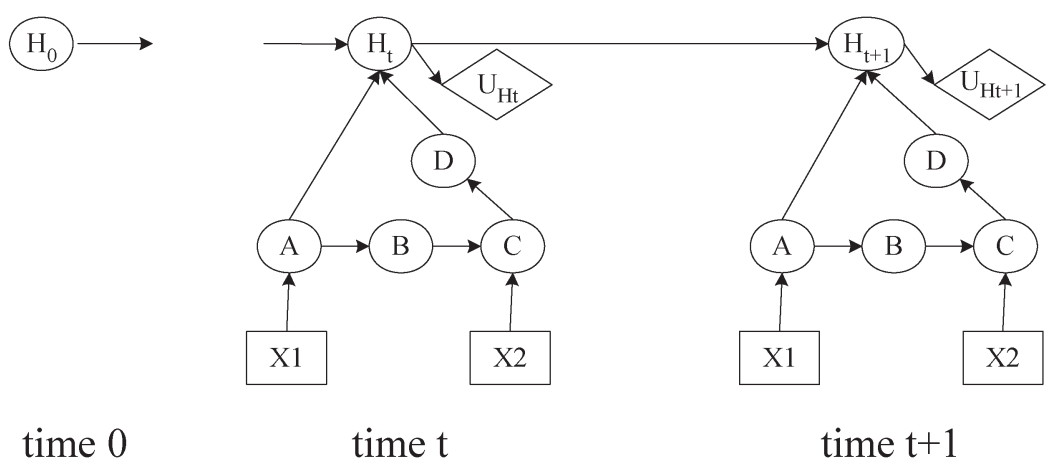

Fig. 2. Dynamic ID model.

than the upstream (i.e., $C_{3} \gg C_{1}+C_{2}$ ), the computational gain is significant.

\section{E. Bounding the Optimal Expected Value}

We show that the interface utilities computed in the factorization approach can be used to derive both an upper and a lower bound of the optimal value of the ID. These bounds have significant implications in practical planning.

We define $f_{\mathrm{in}, U}^{+}$and $f_{\mathrm{in}, U}^{-}$to be the largest and the smallest one among all interface utilities, i.e.,

$$
\begin{aligned}
& f_{\mathrm{in}, U}^{+}=\max _{y_{\mathrm{in}}^{1: m} \in \Omega_{Y_{\mathrm{in}}^{1: m}}} f_{\mathrm{in}, U}\left(y_{\mathrm{in}}^{1: m}\right) \\
& f_{\mathrm{in}, U}^{-}=\min _{y_{\mathrm{in}}^{1: m} \in \Omega_{Y_{\mathrm{in}}^{1: m}}} f_{\mathrm{in}, U}\left(y_{\mathrm{in}}^{1: m}\right)
\end{aligned}
$$

where the max and min are taken over the domain of the variables in $Y_{\mathrm{in}}^{1: m}$. From the theorem, we see that $f_{\mathrm{in}, U}^{+}\left(f_{\mathrm{in}, U}^{-}\right)$ is the upper (lower) bound of the optimal value of the ID. These bounds have significant importance in practice. Suppose, for instance, that these bounds are available to a planner. In one extreme, if the planner expects a utility that is larger than the upper bound, he never bothers to evaluate all the policies and finds the optimal one because even the best policy provides less than he expects. In this case, he needs to redesign the network structure or parameters such that the performance of the ID can be improved. In the other extreme, if the planner expects a utility that is less than the lower bound, again he never bothers to evaluate all the policies and chooses the optimal one because any policy can provide more than he expects. In this case, he can pick any policy and execute it.

We note that from the computational point of view, computing these bounds is easier than evaluating the ID. There are two reasons. First, as discussed earlier, computing these bounds involves only the downstream of the ID, whereas evaluating an ID involves its entire structure. If the downstream contains much fewer variables than the upstream, the interface utilities (and also the bounds) can be obtained efficiently. Second, computing these bounds avoid enumerating all the policies and calculating their expected values.

Finally, the tightness of the bounds depends on the structure of an ID, the CPTs of random nodes, and the value functions of value nodes. It is difficult to characterize a general condition to determine the tightness of the bounds. In our experiments, we empirically show that these bounds are reasonably tight for the tested problems.

\section{EXTENSIONS TO THE FACTORIZATION APPROACH}

In this section, we discuss two extensions to the factorization approach. These extensions deal with reconstructing the policies as the network structure/parameters undergo changes. There are two perspectives. First, at one decision step, the network might change such as more actions being available for a planner's choice, more value nodes needed consideration, and so on. Second, the network might dynamically alter its structure or parameters as time goes by. For example, if a subgoal is successfully accomplished at one step, it can be removed from the network in the subsequent steps.

\section{A. Network Structure/Parameter Changes}

The principle for the factorization approach to accommodate structure or parameter changes is as follows. First, if the changes involve only the upstream of an ID, the interface utilities do not need to be recomputed and can still be shared in evaluating the ID. Specifically, these changes include addition or removal of decision/random/value nodes and also the alternation of CPTs and value functions in the upstream. Second, if the changes involve only the downstream of the ID, the approach needs to reconstruct the interface utilities. Fortunately, the interface probabilities are preserved and the calculations for them can be saved. Third, if the changes involve not only the upstream but also the downstream, the approach needs to recompute both the interface utilities and the interface probabilities.

\section{B. Planning Over Time}

In realistic applications, network parameters may change over time. In this case, we can use a dynamic ID to model the conditional dependencies among nodes over time. In this section, we show how the factorization approach can be used to reconstruct the policies on a step-by-step basis for dynamic IDs.

To facilitate our discussions, we extend the example in Fig. 1 to a dynamic ID. We assume that the variable $H$ evolves over time and let $H_{t}$ denote $H$ at step $t$. The dynamic ID is drawn in Fig. 2. In contrast, the ID in Fig. 1 is said to be static since the multiple decisions are made at one time step. 
The dynamic ID has two prominent features. First, at a single step, the decision problem can be modeled as a static ID. In addition to the nodes and links in Fig. 1, the node $H_{t+1}$ at step $t+1$ has one more parent node $H_{t}$. Second, the intertemporal link between two consecutive nodes carries the historic information about the sequence of performed policies. For step $n+1$, the information can be summarized by a probability distribution of $H_{t}$ conditioned on the history [14].

For a dynamic ID, we are interested in optimal planning on the step-by-step basis. The problem is formulated as: Given an initial probability distribution $P\left(H_{0}\right)$, at step $t+1$, how to efficiently find the policy $\delta\left[=\left(\delta_{1}, \ldots, \delta_{n}\right)\right.$ where $n$ is the number of decision nodes] that maximizes $E_{\delta}\left[U_{H_{t+1}}\right]$ ? To solve the problem, we show: 1) how to select the optimal policy at step $t+1$, given the probability distribution $P\left(H_{t}\right)$; and 2 ) how to sequentially update the probability distribution $P\left(H_{t+1}\right)$ from $P\left(H_{t}\right)$, given a policy $\delta$ at the previous step. After these two questions are settled, we may choose the optimal policy as follows. At step $t+1$, we first choose the optimal policy for the step and then update the probability $P\left(H_{t+1}\right)$ from $P\left(H_{t}\right)$. The procedure repeats at each step.

To answer the first question, we introduce the concept of an augmented interface node and an augmented interface set. We call the node $H_{t}$ an augmented interface node of the ID at step $t+1$ since the node $H_{t}$ can produce impact on the network via altering its probability distribution. In this sense, it is an interface node. ${ }^{3}$ The augmented interface set consists of $\mathcal{Y}_{\text {in }}$ as before and the node $H_{t}$. The downstream of the ID at step $t+1$ remains the same as that of the static ID. Likewise, we may define the two functions $f_{\delta}$ and $f_{\mathrm{in}, U_{H_{t+1}}}$. Therefore, we can use the factorization approach to solve the planning problem over time. The computations involving $f_{\mathrm{in}, U_{H_{t+1}}}$ are factored out. Note that these interface utilities are shared for all policies at each decision step. For the ID in Fig. 2, we can define the following functions for the ID:

$$
\begin{aligned}
& f_{\delta}\left(A, C, H_{t}\right) \\
& \quad=P\left(H_{t}\right) \sum_{B} P\left(A \mid \delta_{1} \delta_{2}\right) P(B \mid A) P\left(C \mid B \delta_{2}\right) \Pi_{i=1}^{2} P_{\delta}\left(X_{i}\right) \\
& f_{\mathrm{in}, U_{H_{t}}}\left(A, C, H_{t}\right) \\
& \quad=\sum_{H_{t+1} D} P\left(H_{t+1} \mid A D H_{t}\right) P(D \mid C) g_{U}\left(H_{t+1}\right) .
\end{aligned}
$$

It can be verified that $E_{\delta}\left[U_{H_{t+1}}\right]=\sum_{A, C, H_{t}} f_{\delta}\left(A, C, H_{t}\right)$. $f_{\text {in }, U_{H_{t}}}\left(A, C, H_{t}\right)$.

To answer the second question, we show how to efficiently compute $P\left(H_{t+1}\right)$, given a distribution $P\left(H_{t}\right)$ and the policy $\delta$ performed at step $t$. We introduce a technique such that the procedure of computing $P\left(H_{t+1}\right)$ can be conducted similar to that of computing $E_{\delta}\left[U_{H_{t+1}}\right]$. Suppose that $H_{t+1}$ can take on two values $h$ (true) and $\neg h$ (false). We first show how to calculate

\footnotetext{
${ }^{3}$ Previously, we defined an interface node to be a node that has parental decision nodes since the choices of decision nodes can affect its CPTs and in turn, the expected value of the ID. In contrast, the node $H_{t}$ is called an augmented interface node since it can change its probability distribution and thus, affect the expected value of the ID.
}

the probability of $H_{t+1}$ being true. Let $V$ be a value node that differs from $U_{H_{t+1}}$ only in its value function. Specifically, $g_{V}$ is 1.0 if its parent $H_{t+1}$ is true; it is 0.0 otherwise. For simplicity, let $H_{t+1}=h(\neg h)$ denote the event that the hypothesis $H_{t+1}$ is true (false). We prove that $E_{\delta}[V]=P_{\delta}\left(H_{t+1}=h\right)$.

Proposition 1: $E_{\delta}[V]=P_{\delta}\left(H_{t+1}=h\right)$. Proof:

$$
\begin{aligned}
E_{\delta}[V]= & \sum_{H_{t+1}} P_{\delta}\left(H_{t+1}\right) g_{V}\left(H_{t+1}\right) \\
= & P_{\delta}\left(H_{t+1}=h\right) g_{V}\left(H_{t+1}=h\right) \\
& +P_{\delta}\left(H_{t+1}=\neg h\right) g_{V}\left(H_{t+1}=\neg h\right) \\
= & P_{\delta}\left(H_{t+1}=h\right) .
\end{aligned}
$$

In the last step, we use the definition of the value function $g_{V}$.

To calculate the probability of $H_{t+1}$ being false, we may define $g_{V}$ as follows: It is 1.0 if its parent $H_{t+1}$ is false; it is 0.0 otherwise. If we define two functions $f_{\delta}$ and $f_{\mathrm{in}, V}$, we see that the computational steps for $E_{\delta}[V]$ are the same as those for computing $E_{\delta}\left[U_{H_{t+1}}\right]$. Hence, computing $P\left(H_{t+1}\right)$ does not add much overhead to ID evaluation.

It is interesting to compare the generic approach and the factorization approach in the context of the dynamic ID. Let the number of decision steps be $T$. Recall that the complexity of computing $f_{\delta}$ is $C_{1}$, the complexity of computing $f_{\mathrm{in}, U_{H_{t+1}}}$ is $C_{3}$, and the complexity of computing $E_{\delta}\left[U_{H_{t+1}}\right]$ is $C_{2}$. Since $C_{1}$ takes constant time, it can be ignored. For one decision step, the factorization approach has the complexity $2^{n} C_{2}+C_{3}$ while the generic approach has the complexity $2^{n}\left(C_{2}+C_{3}\right)$. For $T$ steps, the complexity of the factorization approach is $2^{n} T C_{2}+C_{3}$ [note that this does not include the overhead of computing $P\left(H_{t+1}\right)$ ], while the complexity of the generic approach is $2^{n} T\left(C_{2}+C_{3}\right)$. If $C_{3} \gg C_{2}$, the factorization approach can be extremely efficient.

\section{EXPERIMENTS}

In this section, we report our experiments on both simulation studies and a military planning example. In our experiments, we wrote Matlab-V6.5 codes and ran them on a laptop with a 2.0-GHz central processing unit (CPU) under Windows XP. We compare the factorization approach against the generic bruteforced approach. We chose the generic approach because we were not aware of specific algorithms for evaluating simultaneous IDs. For convenience, we refer to the two algorithms as evalCS (named after computation sharing) and evalBF (named after brute forced).

\section{A. Simulation Studies}

To thoroughly evaluate the performance of the factorization approach, we conducted simulated studies on the ID in the left chart of Fig. 3, which is similar to the military planning examples in [15]. It is referred to as the static ID in the rest of this section. The CPTs are randomly generated. The value functions for value nodes are manually specified.

Specifically, our experiments are designed to: 1) evaluate the performances of the factorization approach for static and 

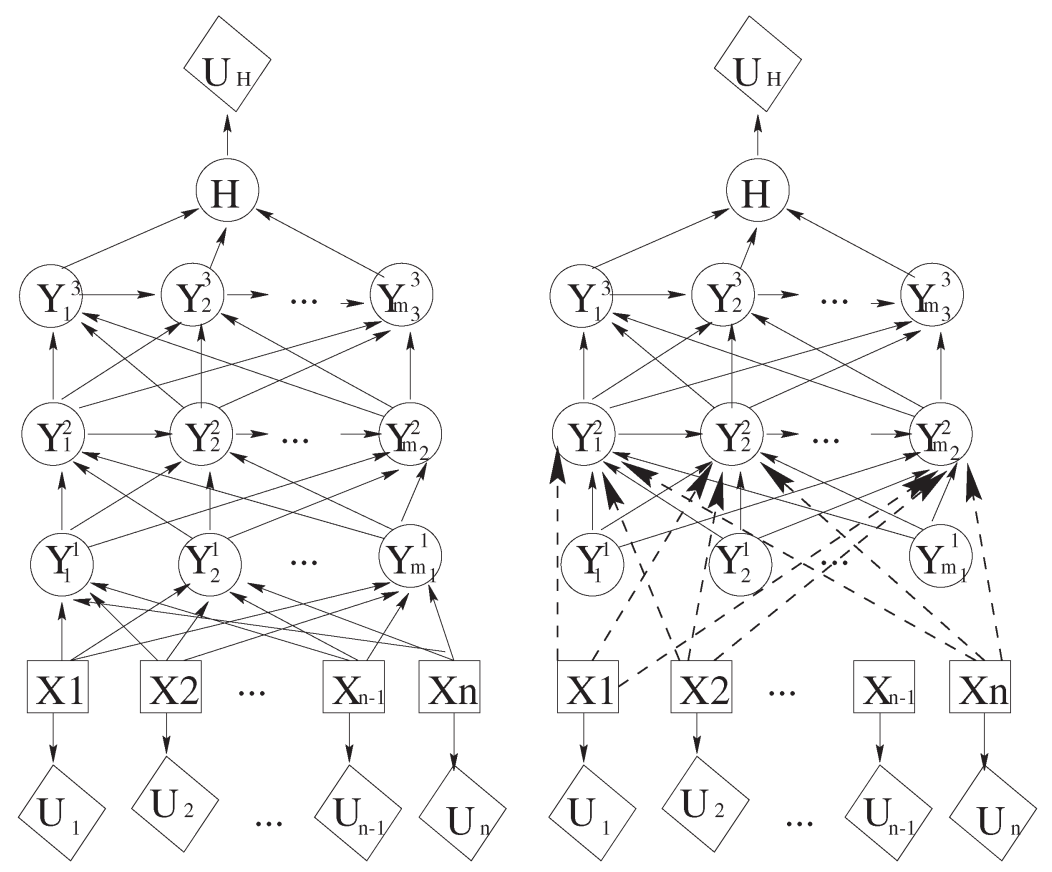

Fig. 3. Test example is shown in the left chart, while the right is its variant for comparative studies.
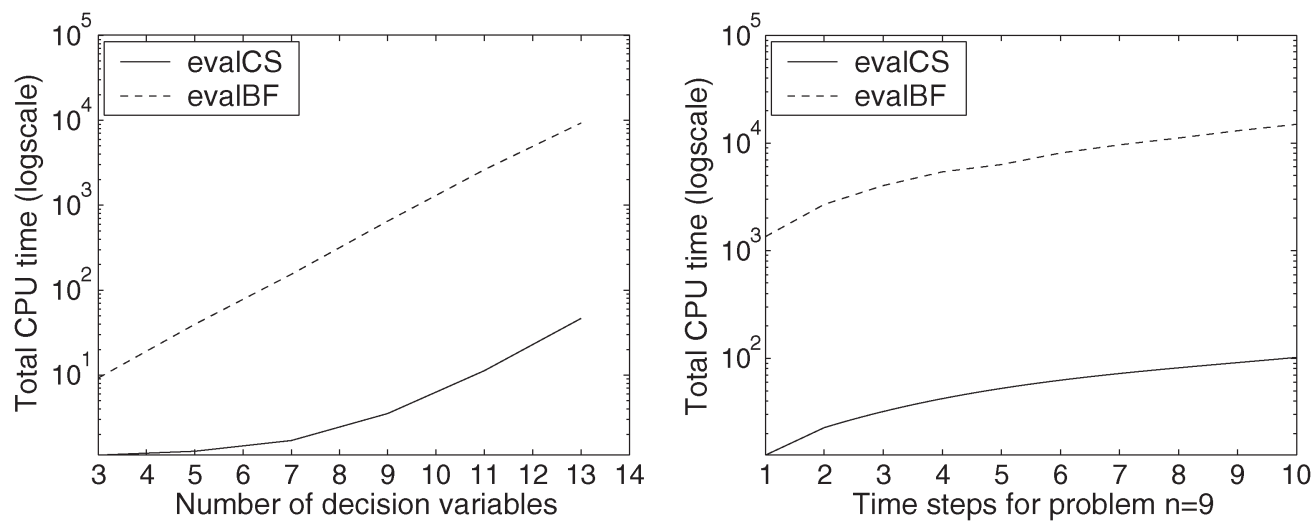

Fig. 4. Performance comparison of evalBF and evalCS.

dynamic IDs; 2) show the tightness of bounds derived from the interface utilities; 3) demonstrate how the computational gain achieved by the approach varies with different network structures; and 4) demonstrate the computational gain by adapting the approach to account for newly added decisions and value nodes.

1) Performances of the Factorization Approach: To see how the performances of the algorithms vary with the number of decision nodes, we fix the number of random nodes at each level at four and vary the number of decision nodes. Thus, the static ID with $n$ decision nodes has additionally ten random nodes and $n+1$ value nodes. We ran evalBF and evalCS for seven problems with $n=3,5, \ldots, 13$. The timing data are presented in the left chart of Fig. 4. The chart gives the total CPU seconds that the algorithms took for each of the problems. Note that the vertical direction is drawn in $\log$ scale. The solid (dashed) curve is for evalCS (evalBF). It can be seen that evalCS is considerably more efficient than evalBF. For instance, from our data, for $n=9$, to evaluate 512 policies,
evalCS took $3.51 \mathrm{~s}$ while evalBF, $646.74 \mathrm{~s}$; for $n=13$, to evaluate 8192 policies, evalCS used $46.32 \mathrm{~s}$ while evalBF, $9284.05 \mathrm{~s}$.

To quantitatively characterize how much savings the factorization procedure can bring about, we use the timing results of evalCS to predict the performance of evalBF. Recall that the complexity of evaluating a policy breaks into three fractions $C_{1}, C_{3}$, and $C_{2}$. We ignore $C_{1}$ since it is a constant. For each problem, we estimate $C_{3}$ by the actual seconds $\hat{C}_{3}$ of computing $f_{\mathrm{in}, U_{H}}$, and $C_{2}$ by $\hat{C}_{2}$ as (the total CPU time $\left.\hat{C}_{3}\right) /($ the number of policies). The complexity of evalBF is predicted by $2^{n}\left(\hat{C}_{2}+\hat{C}_{3}\right)$. We found that these estimations are almost the same as the actual timing results of evalBF. This suggests the effectiveness of our complexity analysis.

We also tested the algorithms over the dynamic ID in Fig. 5, which is an extension of the left chart of Fig. 3.

Initially, the probability of the node $H$ being false is set to 1.0. Its probability is updated at each decision step. We ran both algorithms for up to ten decision steps. We showed the 


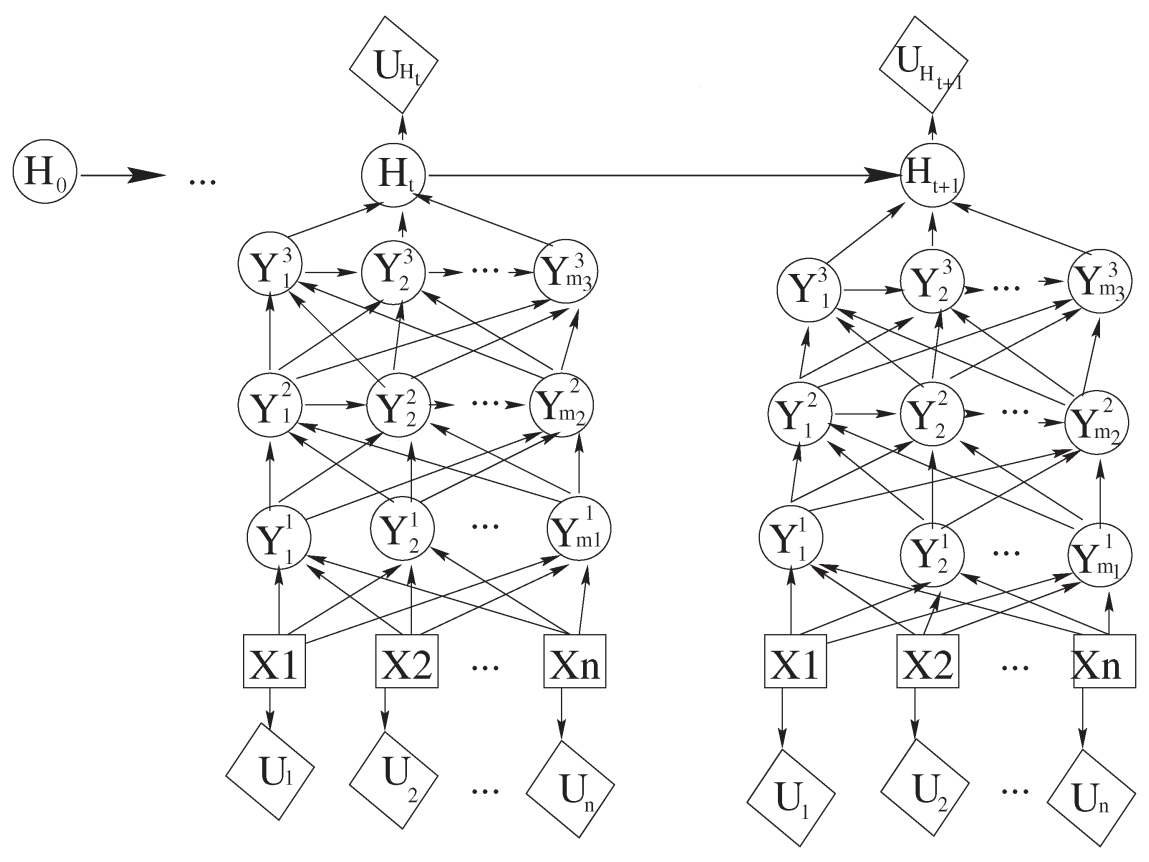

Fig. 5. Dynamic influence diagram.

total CPU time for the ID with nine decision nodes in the right chart of Fig. 4. The chart gives the total CPU seconds for both algorithms against the time steps. Note that again the vertical direction is drawn in log scale. It can be seen that the CPU time linearly increases with the elapsed time for evalBF while its increase is negligible for evalCS. This is not a surprising observation. In evalBF, all policies are evaluated at each step. The time cost for all steps remains the same. Hence, the increase is linear. From our data, evalBF uses about $1300 \mathrm{~s}$ to evaluate all $512\left(2^{9}\right)$ policies at each step. However, in evalCS, the interface utilities are computed only once at the first step. So, we observe that at the first step, evalCS takes about $2.66 \mathrm{~s}$ to compute these utilities; thereafter, each step takes about only $10 \mathrm{~s}$ to evaluate all 512 policies. The increase is negligible when compared against that in evalBF.

2) Tightness of Bounds: To show the tightness of the upper and lower bounds of the optimal expected value, in Fig. 6, we plot the optimal value (the middle curve) and these bounds (the upper and lower curves) for the static IDs with 3, 5, ..,13 decision nodes. We see that these bounds are reasonably tight for the tested problems. For example, for $n=7$, the optimal value is 871.47 while the bounds are 722.91 and 936.637. Although it is difficult to quantitatively analyze the properties of these bounds, these experiments show they can be tight at least for these tested problems.

3) Computational Gain Under Network-Structure Changes: To demonstrate how the computational gain of evalCS varies with different network structures, we run evalCS over the static ID and a modified version of it. The modified ID is obtained as follows: Every link from $X_{i}$ to $Y_{j}^{1}$ is redirected to $Y_{j}^{2}$. The resulting ID is shown in the right chart of Fig. 3 . Its upstream is $\mathcal{X} \cup Y_{1: m_{1}}^{1} \cup Y_{1: m_{2}}^{2} \cup U_{1: n}$, whereas its downstream is $Y_{1: m_{3}}^{3} \cup\{H\} \cup\left\{U_{H}\right\}$, where $U_{1: n}$ means the set of value nodes, and $Y_{1: m_{i}}^{i}$ means the set of nodes $Y_{j}^{i}$, i.e., $Y_{1: m_{i}}^{i}=\left\{Y_{1}^{i}, \ldots, Y_{m_{i}}^{i}\right\}$ for $i=1,2,3$. Compared with that of

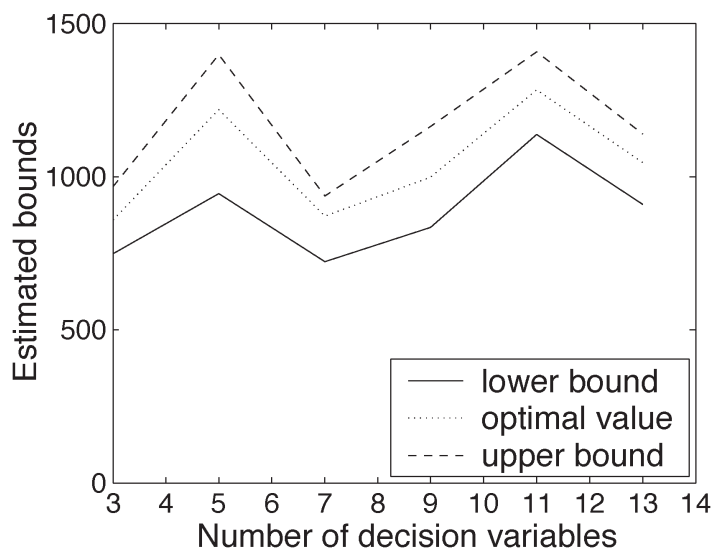

Fig. 6. Lower and upper bounds obtained from the interface utilities.

the static ID, the downstream of the modified ID contains fewer random nodes. We expect: 1) evalCS is still more efficient than evalBF in the modified ID, since its downstream contains a number of random nodes; and 2) evalCS achieves less savings in modified ID than it does in the original ID.

The experiments presented in Fig. 7 confirm these expectations. First, the left chart plots the CPU seconds (in log scale) that evalCS and evalBF take for the modified IDs with a different number of decision variables. It can be seen that evalCS is more efficient. Second, the right chart plots the magnitudes of the savings brought by evalCS. For each approach, the saving magnitude is measured by the quotient of the total time of evalBF and that of evalCS. The magnitudes are drawn in the vertical direction. For a modified ID, evalCS is about 14 times faster than evalBF. For the original IDs, the magnitudes vary with the number of their decision nodes. We see that the computational savings brought by evalCS are more significant for IDs whose downstream contains more nodes. 

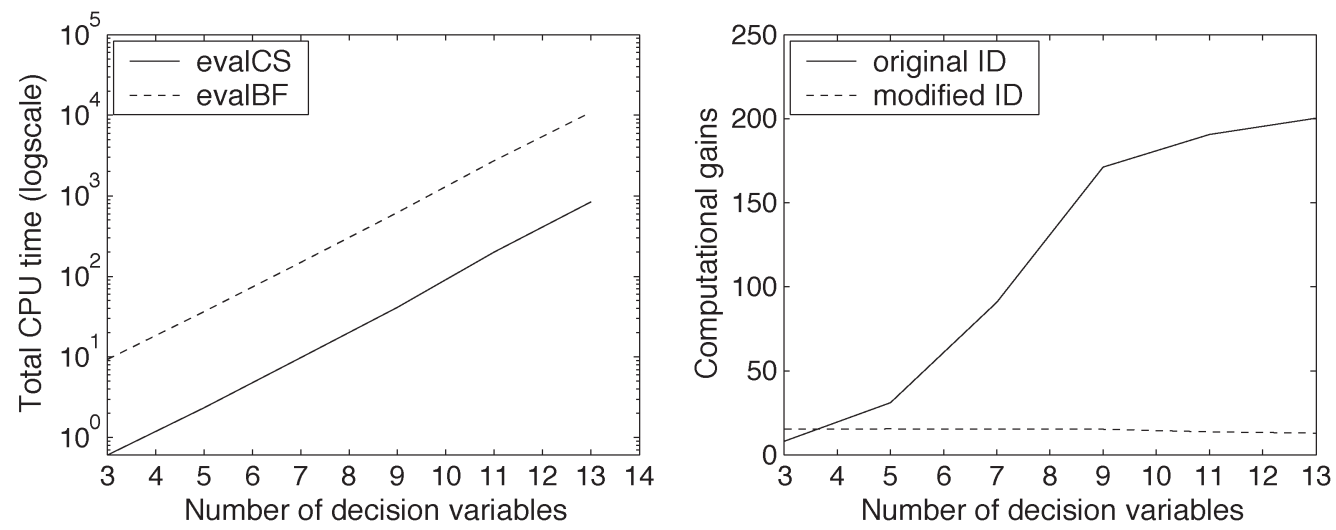

Fig. 7. Computational gains versus network structure.
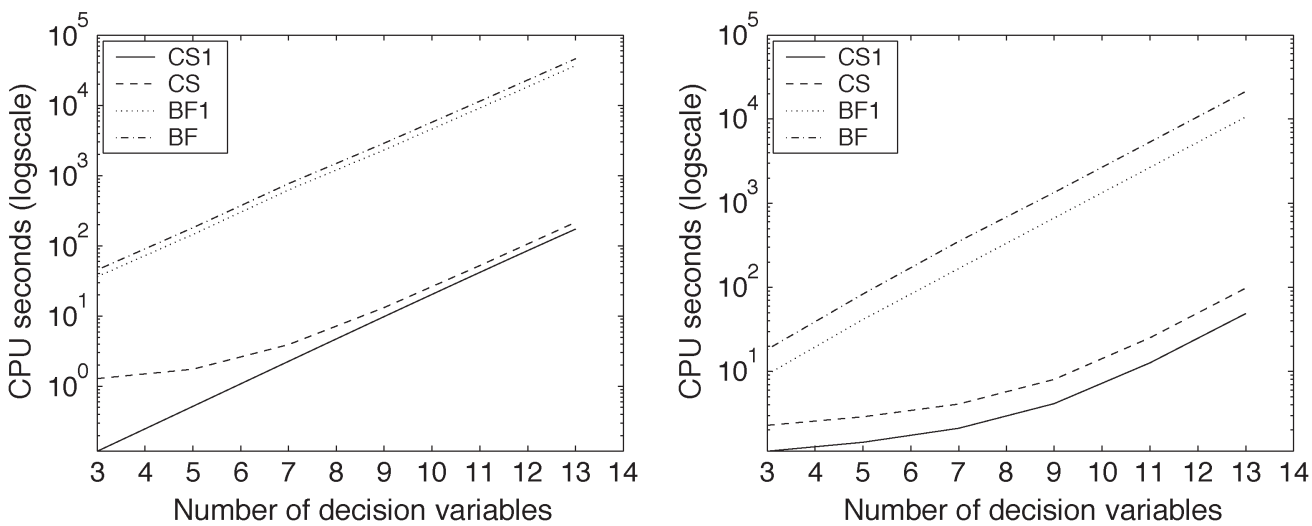

Fig. 8. Replanning for added action/value nodes. In both charts, the curves from the top and bottom plot the total/replanning time for the generic approach, and the total/replanning time for the factorization approach.

4) Computational Gain Under Network-Parameter Changes: We also conducted experiments to show how the factorization approach achieves computational savings as the network changes. For this purpose, given the static ID, we first evaluate it (the planning phase), then add more nodes to the ID and reevaluate it (the replanning phase). We like to compare both the replanning time and total time of the factorization approach against that of the generic approach.

In one experiment, we first evaluate a static ID with $n$ decision nodes. We then add two decision nodes to the ID and evaluate the modified ID. Every newly added node has a link from itself to every $Y_{j}^{1}$ node. The timing results in log scale are presented in the left chart of Fig. 8. In the chart, the curve corresponding to $\mathrm{CS} 1(\mathrm{BF} 1)$ depicts the replanning time for the factorization (generic) approach, whereas the curve corresponding to CS (BF) depicts the total time similarly. We see that for the tested problems, the factorization approach achieves considerable savings in replanning when more decision nodes are added. For instance, for the ID with nine decision nodes, the factorization and generic approach, respectively, takes 9.80 and $2313.94 \mathrm{~s}$. These savings are achieved through sharing the interface utilities computed during the evaluation of the original ID. Since the factorization approach takes much less time in both evaluating and reevaluating the ID, its total time is considerably less than that used by the generic approach.

In the other experiment, we evaluate the ID and then add one more value node for replanning. The added value node has a link from every node $Y_{j}^{2}$ for $j=1, \ldots, 4$. In computing the expected value of the added value node, we still use the factorization approach. In reevaluating an ID, we do not recompute the expected value of the existing value node. The timing results in log scale are collected in the right chart of Fig. 8. The legends read similar to those in the left chart. It can be seen that the factorization approach can achieve great savings in replanning. The reason is obvious: The factorized computations are saved in computing the expected value of the newly added value node. By taking advantage of shared computations in evaluating two value nodes, the total time used by the factorization approach is considerably less than that by the generic approach.

\section{B. A Military Planning Example}

We applied the factorization approach to a hypothetical military planning example, which is illustrated in Fig. 9. The overall military goal is to win a war or to bring a tyrant to justice. The goal is represented by a Hypothesis node, which is on the top of the figure. There are 12 primitive actions, namely destroy_C2, destroy_Radars, ..., operate_special_force, which are on the bottom side. Performing an action has direct effects of specific purpose. For instance, if the action destroy_Radars is performed, the EW/GCIRadars is destroyed with a high probability. These effects alter the overall goal through altering the low-level subgoals. For instance, the status 


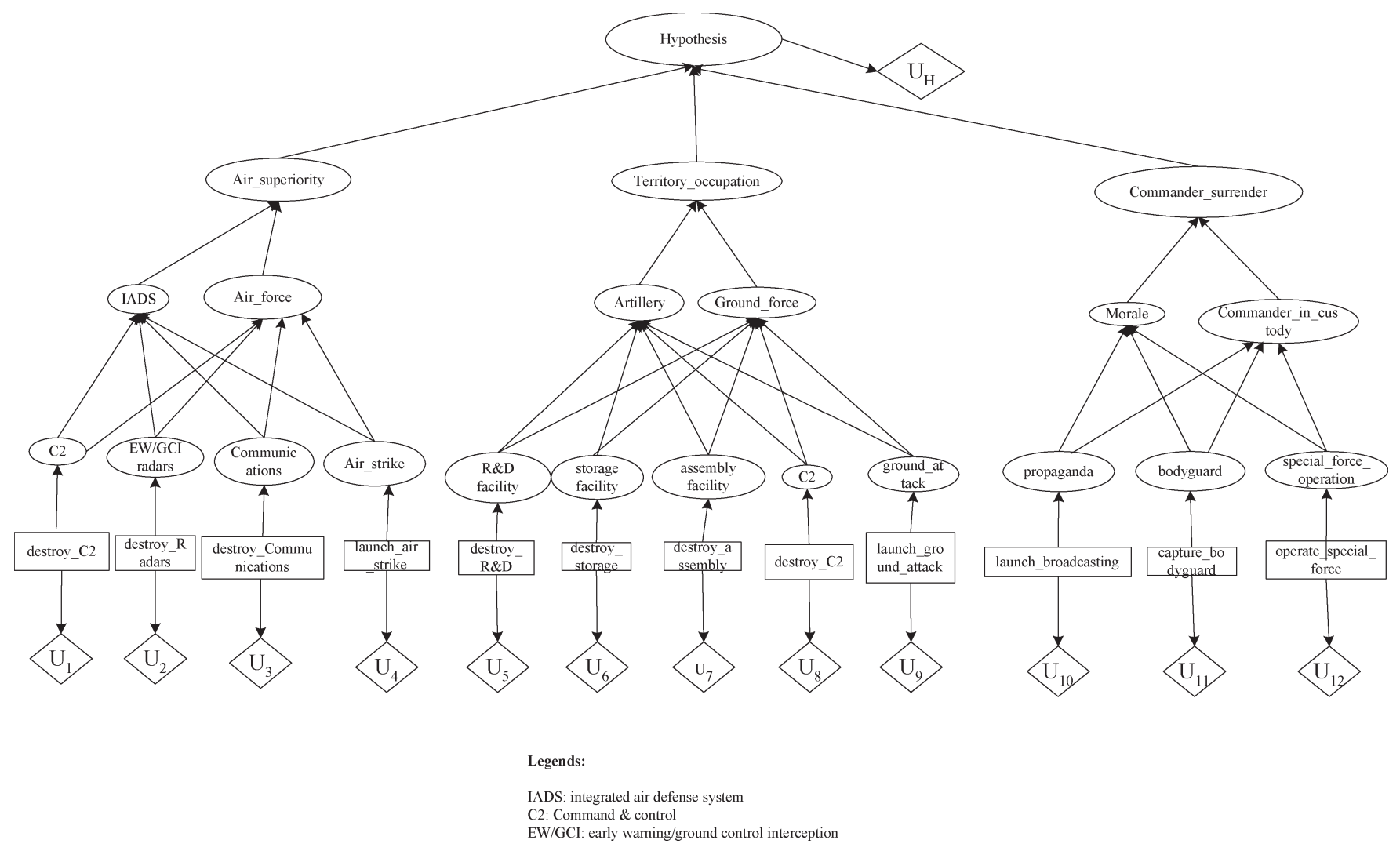

Fig. 9. Static ID illustrating a military planning problem.

of C2 (command and control), EW/GCIRadars and Communications facilities, and Air strike determine the workability of the integrated air defense system (IADS) and the strength of the enemy air force. In turn, the workability of IADS system and the strength of the enemy air force determine the loss of Air_superiority. The Air_superiority, Territory_occupation, and Commander_surrender are three subgoals determining the overall goal success. Without loss of generality, we assume all nodes are binary. In the example, each decision node is associated with a value node encoding the cost of performing the action, and the hypothesis node is associated with a value node encoding the utility of goal success. The optimal policy needs to balance the utility of goal success and cost of performing actions.

We designed a reasonable set of CPTs and value functions. For the Hypothesis node, if all the subgoals Air_superiority, Territory_occupation, and Command_surrender are achieved, the overall goal is successfully achieved. If one of the subgoals is to be achieved, the probability of the overall success is decreased by 0.3 ; however, if none of the subgoals is achieved, the overall goal fails with certainty. Similarly, for the subgoal Air_superiority, the two influencing factors are IADS and Air force. If the IADS system works well and Air force is strong, Air superiority is true for the enemy air force; if either the IADS system works poorly or Air force is weak, the probability of Air superiority being true is decreased by 0.5 . Other CPTs for
Territory occupation and Command surrender are set analogously to those for Air superiority. A similar strategy is used in parameterizing the nodes IADS, Air_force, Artillery, Ground_force, Morale, and Commander_in_custody. In determining the CPTs for random nodes that are immediate children of the decision nodes, we assume that an action achieves its intended effect with probability 0.9. For example, a destroy_Radars decision will destroy the EW/GCI radars with probability 0.9 . To complete the ID definition, we also assigned value functions. If the goal is successfully achieved, the reward is 1000; otherwise, the cost, i.e., a negative reward, is 500. For other decision nodes, if a ground attack is launched, the cost is 150 ; if the special force operation is performed, its cost is 100; if the commander decides to capture the bodyguards of the tyrant, the operating cost is 80 ; if an air strike is launched, the cost is 50; for any other actions, their operating cost is 20 .

Our primary interest is in the performance of the factorization algorithm. From our data, to evaluate the ID, the factorization algorithm took $45 \mathrm{~s}$, while the brute-forced algorithm took 9012 s. Hence, the computational saving is tremendous. We can explain the performance difference by the ID structure-its downstream contains a large number of nodes: all random nodes and the value node associated with the goal. Since its downstream contains far more random nodes than its upstream, the approach is expected to be significantly more efficient. Our secondary interest is concerned with the optimal policy. The optimal policy is the one that performs only air strike and special force operation. The expected value of the ID 
is 561.98 , and the probability of goal success is 0.81 . We note that the optimal policy excludes "launching a ground attack," although it is the action that is most likely to lead to goal success. One possible reason, as explained earlier, is that the action is excluded due to the high operating cost of performing the action.

\section{CONCLUSION AND FUTURE WORK}

In this paper, we studied a special ID class, namely simultaneous IDs, where multiple decisions need to be made at one time step. We intended to make two contributions. First, we examined a simultaneous ID and studied its theoretical properties. We showed that such an ID can be decomposed into an upstream fraction and a downstream fraction, and that the expected value of a value node under a policy can be represented as the sum of interface utilities that involve only the downstream fraction, weighted by the corresponding interface probabilities that involve only the upstream fraction. The interface utilities naturally provide an upper and lower bound of the optimal value of the ID. Second, we proposed a novel factorization algorithm to evaluate a simultaneous ID. The interface utilities are independent of the individual policies; therefore, they can be calculated once but used across all policies in evaluating them. We also extend the factorization approach to a dynamic ID. The algorithm has been tested on simulation studies and a military planning example. Our experiments showed that the factorization algorithm is significantly more efficient than the generic algorithm in evaluating a simultaneous ID.

To further speed up ID evaluating, one future direction is to combine the factorization approach with the approaches of reducing the search space. In this paper, we address one difficulty in ID evaluation, i.e., evaluating individual policies. Another difficulty in ID evaluation is that the policy space contains exponentially many polices and one needs to evaluate all of them in order to find the optimal one. The ID evaluation process can be accelerated if the technique in this paper can be integrated with the approaches of reducing the search space.

\section{ACKNOWLEDGMENT}

The authors would like to thank J. Lemmer, D. Gossink, and J. Dussault from AFRL/Rome for introducing the problem to us and for numerous technical exchanges on the related issues. The authors are grateful to three anonymous reviewers for their insightful comments in improving the paper. The authors are also grateful to W. Liao and Z. Zhu for insightful discussions.

\section{REFERENCES}

[1] R. Howard and J. Matheson, "Influence diagrams," in The Principles and Applications of Decision Analysis, R. Howard and J. Matheson, Eds. Menlo Park, CA: Strategic Decisions Group, 1984, pp. 719-762.

[2] H. Raiffa, Decision Analysis. Reading, MA: Addison-Wesley, 1968.

[3] R. D. Shachter, "Evaluating influence diagrams," Oper. Res., vol. 34, no. 6, pp. 871-882, 1986.

[4] P. P. Shenoy, "Valuation-based systems for Bayesian decision analysis," Oper. Res., vol. 40, no. 3, pp. 463-484, 1992.

[5] F. Jensen, F. V. Jensen, and S. L. Dittmer, "From influence diagram to junction trees," in Proc. 10th Conf. Uncertainty Artificial Intelligence, Seattle, WA, 1994, pp. 367-373.
[6] R. Qi and D. Poole, "A new method for influence diagram evaluation," Comput. Intell., vol. 11, no. 1, pp. 1-34, 1995.

[7] N. L. Zhang and D. Poole, "Stepwise-decomposable influence diagram," in Proc. 3rd Int. Conf. Principles Knowledge Representation and Reasoning, Cambridge, MA, 1992, pp. 141-152.

[8] G. F. Cooper, "A method for using belief networks as influence diagrams," in Proc. 4th Workshop Uncertainty Artificial Intelligence, St. Paul, MN, 1988, pp. 55-63.

[9] R. Shachter and M. Peot, "Decision making using probabilistic inference methods," in Proc. 8th Annu. Conf. Uncertainty Artificial Intelligence (UAI), Stanford, CA. San Mateo, CA: Morgan Kaufmann, 1992, pp. 276-283.

[10] T. Nielsen and F. Jensen, "Well-defined decision scenarios," in Proc. 15th Conf. Uncertainty Artificial Intelligence, Stockholm, Sweden, 1999, pp. 502-511.

[11] F. Jensen and M. Vomlelova, "Unconstrained influence diagrams," in Proc. 18th Conf. Uncertainty Artificial Intelligence, Edmonton, AB, Canada, 2002, pp. 234-241.

[12] S. L. Lauritzen and D. Nilsson, "Representing and solving decision problems with limited information," Manage. Sci., vol. 47, no. 9, pp. 1238-1251, 2001.

[13] N. L. Zhang, "Probabilistic inferences in influence diagrams," in Proc. 14th Conf. Uncertainty Artificial Intelligence, Madison, WI, 1998, pp. 514-522.

[14] K. J. Aström, "Optimal control of Markov decision processes with incomplete state estimation," J. Math. Anal. Appl., vol. 10, no. 3, pp. 174-205, 1965.

[15] U. Kuter, D. Nau, and J. F. Lemmer, "Interactive planning under uncertainty with causal modeling and analysis," Dept. Comput. Sci., Univ. Maryland, College Park, Tech. Rep. CS-TR-4434, 2003.

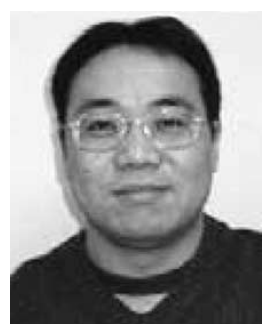

Weihong Zhang received the Ph.D. degree in computer science from The Hong Kong University of Science and Technology, Kowloon, Hong Kong, in 2001.

He then worked as a Postdoc Researcher with the Department of Computer Science and Engineering, Washington University, Saint Louis, MO. He is currently a Postdoc Researcher with the Department of Electrical, Computer, and Systems Engineering, Rensselaer Polytechnic Institute, Troy, NY. He has conducted research in artificial intelligence, probabilistic inferences and decision making under uncertainty, graphical models and their applications in sensor networks and human-computer interaction. He has published more than 10 papers in peer-reviewed journals and conferences.

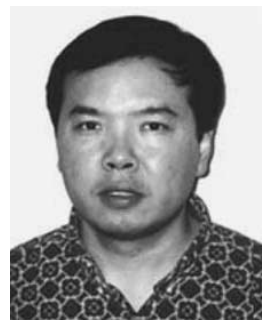

Qiang Ji (S'92-M'98-SM'04) received the Ph.D. degree in electrical engineering from the University of Washington in 1998.

$\mathrm{He}$ is currently an Associate Professor with the Department of Electrical, Computer, and Systems Engineering at Rensselaer Polytechnic Institute, Troy, NY. His areas of research include computer vision, probabilistic reasoning for decision making and information fusion, pattern recognition, and robotics. He has published more than 70 papers in peer-reviewed journals and conferences. His research has been funded by local and federal government agencies including National Science Foundation (NSF), National Institutes of Health (NIH), Air Force Office of Scientific Research (AFOSR), Office of Naval Research (ONR), Defense Advanced Research Projects Agency (DARPA), and Army Research Office (ARO) and by private companies including Boeing and Honda. His latest research focuses on face detection and recognition, facial-expression analysis, image segmentation, object tracking, user affect modeling and recognition, and active information fusion for decision making under uncertainty. 